\title{
Dynamic Traffic Congestion Simulation and Dissipation Control Based on Traffic Flow Theory Model and Neural Network Data Calibration Algorithm
}

\author{
Li Wang, ${ }^{1}$ Shimin Lin, ${ }^{2}$ Jingfeng Yang, ${ }^{3,4}$ Nanfeng Zhang, ${ }^{5}$ Ji Yang, ${ }^{3}$ Yong Li, \\ Handong Zhou, ${ }^{4}$ Feng Yang, ${ }^{2,3}$ and Zhifu $\mathrm{Li}^{6}$ \\ ${ }^{1}$ School of Computer Software, Tianjin University, Tianjin 300072, China \\ ${ }^{2}$ Department of Computer Science and Engineering, Faculty of Engineering, The Chinese University of Hong Kong, \\ Shatin 999077, Hong Kong \\ ${ }^{3}$ Open Laboratory of Geo-Spatial Information Technology and Application of Guangdong Province, Guangzhou Institute of Geography, \\ Guangzhou 510070, China \\ ${ }^{4}$ Guangzhou Yuntu Information Technology Co., Ltd., Guangzhou 510665, China \\ ${ }^{5}$ Guangzhou Entry-Exit Inspection and Quarantine Bureau, Guangzhou 510623, China \\ ${ }^{6}$ School of Mechanical and Electrical Engineering, Guangzhou University, Guangzhou 510006, China
}

Correspondence should be addressed to Jingfeng Yang; jingfengyang@126.com and Zhifu Li; sundylzf@gmail.com

Received 30 June 2017; Accepted 16 October 2017; Published 31 December 2017

Academic Editor: Junpei Zhong

Copyright (C) 2017 Li Wang et al. This is an open access article distributed under the Creative Commons Attribution License, which permits unrestricted use, distribution, and reproduction in any medium, provided the original work is properly cited.

\begin{abstract}
Traffic congestion is a common problem in many countries, especially in big cities. At present, China's urban road traffic accidents occur frequently, the occurrence frequency is high, the accident causes traffic congestion, and accidents cause traffic congestion and vice versa. The occurrence of traffic accidents usually leads to the reduction of road traffic capacity and the formation of traffic bottlenecks, causing the traffic congestion. In this paper, the formation and propagation of traffic congestion are simulated by using the improved medium traffic model, and the control strategy of congestion dissipation is studied. From the point of view of quantitative traffic congestion, the paper provides the fact that the simulation platform of urban traffic integration is constructed, and a feasible data analysis, learning, and parameter calibration method based on RBF neural network is proposed, which is used to determine the corresponding decision support system. The simulation results prove that the control strategy proposed in this paper is effective and feasible. According to the temporal and spatial evolution of the paper, we can see that the network has been improved on the whole.
\end{abstract}

\section{Introduction}

Traffic congestion is a common problem in many countries, especially in big cities. As far as our country is concerned, it is gradually spread to the medium cities and even small cities. This has brought a huge negative impact on the economic and social development of the region. For travelers, traffic congestion will lead to increased travel time, travel costs, and the quality of travel. From the point of view of traffic management, traffic congestion will result in higher operating costs and low efficiency of traffic network. At the social level, traffic congestion will cause traffic accidents, air pollution, noise pollution, and other environmental issues. Research on the formation and propagation of traffic congestion is a new technology to control the traffic jam. The sources of traffic congestion can be summarized into three categories: (1) the temporary barrier; (2) the bottleneck of the network's capacity which is the bottleneck; (3) the random fluctuation of a particular region in the network. In this paper, traffic congestion under accident conditions is the first kind of traffic congestion caused by traffic congestion. All known and unknown events affecting the road traffic flow are collectively referred to as traffic accidents [1]. At present, China's urban road traffic accidents occur frequently, the 
occurrence frequency is high, the accident causes traffic congestion, and accidents cause traffic congestion and vice versa. The occurrence of traffic accidents usually leads to the reduction of road traffic capacity and the formation of traffic bottlenecks, causing the traffic congestion. If the accident cannot be cleared in time, the vehicle will be up to the upper section of the line after the queue. If there is no timely evacuation, traffic congestion will quickly spread on the network, so as to increase the delay time of the traveler. Studies have shown that exiting road canalization is unreasonable, it is easy to form closed-loop congestion phenomenon in the dissipation of congestion. Congestion closed loop is the formation of the queue of the tail back to the congestion of the starting point. Once a closed loop is formed, traffic congestion itself is difficult to dissipate in the absence of artificial intervention [2].

In this paper, the formation and propagation of traffic congestion are simulated by using the improved medium traffic model, and the control strategy of congestion dissipation is studied. As the traffic congestion is mainly caused by accidents, temporary traffic control measures are the prohibition of the left, the prohibition of the right, and the right to ban. Vehicle ban measures can make originally lined up waiting vehicle restarted and select the way leaving the congested area. More important, timely application of vehicle turn prohibition measures can effectively avoid the formation of closed-loop congestion; even under conditions of closedloop congestion vehicles are prohibited from turning to relieve the closed-loop congestion, so as to avoid congestion of malignant spread [3].

From the point of view of quantitative traffic congestion, the paper provides the fact that the simulation platform of urban traffic integration is constructed and a feasible data analysis method is proposed, which is used to determine the corresponding decision support system. The model is based on the international application of extensive and sophisticated series of software, which is based on learning and parameter calibration [4-11], optimization of macro simulation model, maintenance, macro (network), and medium (node and intersection). Through the platform and model, it forms the traffic state analysis of data support and auxiliary analysis platform, improving the urban road traffic forecasting ability, to provide the necessary technical support for the establishment of the urban project construction traffic impact assessment system.

\section{Traffic Flow Theory Model and Data Calibration Algorithm}

2.1. Traffic Flow Theory Introduction. Different traffic models can play a role in different aspects. From space, the macro traffic model can be used to analyze the main road network in the whole area, and the medium traffic model can be used to analyze a certain local project area, which can be used to support traffic planning and traffic engineering. In time, the macro traffic model takes a day or a peak hour as the scale; the medium traffic model is built on a smaller scale (e.g., every hour, every 15 minutes, and every minute); and the microscopic traffic simulation model can be fine to the second unit (e.g., every second, every 0.1 seconds). The medium traffic model is based on the four steps of traffic planning in the macroscopic traffic model. By using the information of the basic road network, the traffic demand information is updated and the traffic assignment method is used to achieve the traffic model to express the actual state of the road network. Medium traffic model is more detailed, in addition to primary and secondary roads and main roads, and it also includes a small road. In the medium traffic model, it is necessary to take account of its drainage and traffic control methods and to consider the signal timing scheme for signal control intersection. Medium traffic model is the second level of traffic research as a supplementary tool, direct simulation of the macro background, the development and adjustment of the land, the layout of the land, and the improvement of the regional transportation system.

The micro model reflects and simulates the operation process of a single vehicle in the road network, directly simulating the operation of traffic guidance and signal control. The microscopic traffic simulation model is further refined to a number of intersections, and the parameters of each lane are set up, which can be analyzed in detail. In time, the microscopic traffic simulation model can be refined to a second as a unit (e.g., every 0.1 seconds). As the auxiliary tool of the third levels of traffic research, the model of the onlookers is analyzed and compared with the indexes of the vehicle running process, and the detailed design scheme of the local traffic improvement measures and signal optimization measures are evaluated and analyzed [7]. From the perspective of traffic demand and traffic supply, the medium traffic model is to analyze the causes of traffic congestion and to analyze the congestion control scheme.

2.2. Meso Traffic Dynamics Model. In this study, the Zhuzilin area is used to establish an example of a mesoscopic traffic model for congestion cause analysis and to make recommendations for improvement, comparison, and analysis of schemes. As this project is mainly integrated model of the technical analysis method research, the results may be different from the actual traffic conditions.

The traffic flow theory model can be divided into macro and micro methods. In view of the dynamic model, the dynamic model can meet the requirements of accuracy and has good real-time performance, so it is a hot spot in the field of intelligent transportation.

2.2.1. Queuing Theory. According to the queuing theory, the queuing delay of the queue in the $i$ car is

$$
\frac{i}{c}
$$

which $c$ represents lane group output capacity (such as vehicle queue dissipation rate; traffic control signal influence on traffic flow can reflect on the change of $C$ ); $c t$ is number of vehicles for departure time $t$; if a mobile vehicle at time $t$ reached the tail of the queue, its position is

$$
q(t)=q(0)+l(c t-m),
$$



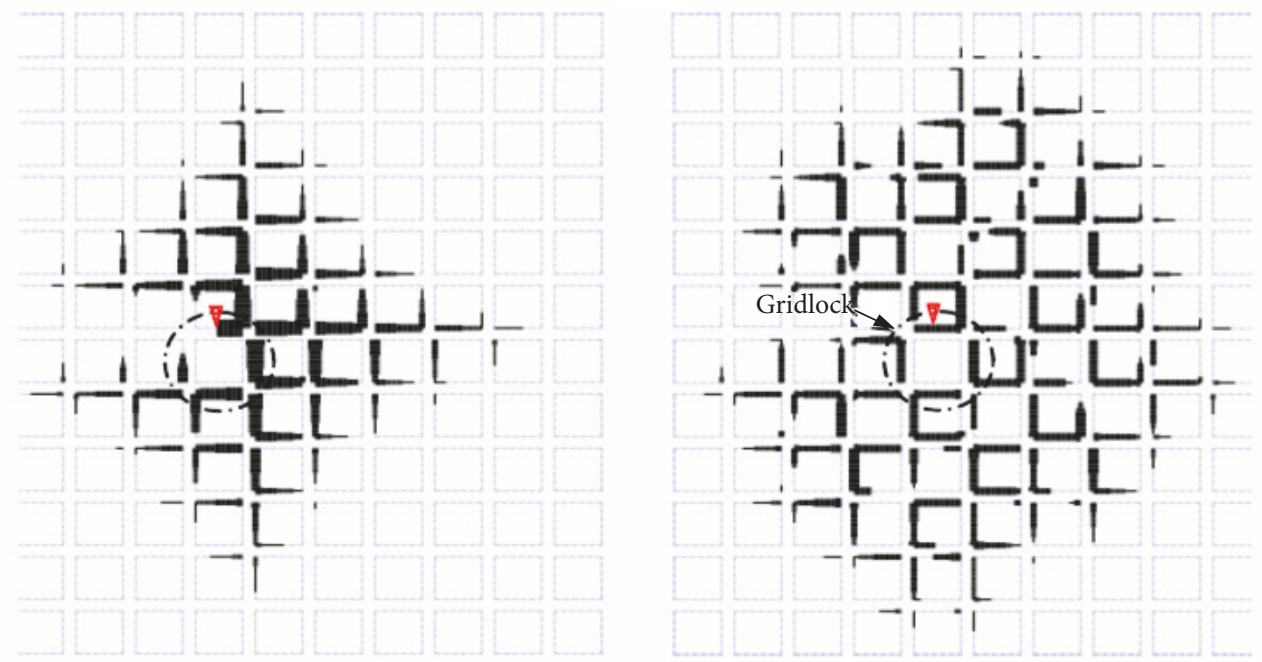

$\nabla$ Incident position

FIgURE 1: Traffic congestion.

where $q(0)$ is the location; the end of the time $t=0 t=0$, and $l$ from 1 jam is the average length of the vehicle. $l=1 / \rho_{\text {jam }}$ Jam is crowded density; $m$ is the number of vehicles in time $t=0 t$ between the inspection of vehicles and the end of the queue of vehicles running. In fact, the number of vehicles entering the queue before the vehicle is considered.

It is worth noting that the applicable conditions of the model are

$$
0<q(t)<L
$$

2.2.2. Speed Model. In the medium traffic simulator, the traffic network is divided into the connecting line, the node, and the loading point. Each connecting line is divided into a plurality of segments, each segment containing a moving element and a queue element $[12,13]$. The speed of the vehicle in the moving element is determined by the velocity density model. The model is based on the assumption that the upstream part of the section is kept constant, the downstream part contains a deceleration area, and the speed of the vehicle in the region varies linearly with the position (as shown in Figure 1), where $v_{u}$ is the speed of the end of the section of the section, $v_{a}$ is the speed of the end of the section of the section, and $L_{s}$ is the length of the deceleration area. $L_{s}$ is related to the terrain features and traffic conditions of the section.

The upstream end of the road is set at 0 points, and the length of the section is $L$, then the downstream end of the road is located at the position of $L$, which establishes the relationship between the speed and position

$$
\begin{gathered}
v(z)= \begin{cases}v_{u}, & 0 \leq z \leq L-L_{s}, \\
\lambda(z-L)+v_{d}, & L-L_{s} \leq z,\end{cases} \\
\lambda=\frac{v_{d}-v_{u}}{L_{s}} .
\end{gathered}
$$

If there is no queuing phenomenon, the speed at the downstream end of the section is affected by the traffic conditions of the lower section of the section. If there is a vehicle queue phenomenon in the section, the speed of the lower end of the section is determined by the line dissipation rate (i.e., the output capacity). The velocity $v_{u}$ of the upstream end of the segment is a function of the average density of the segment moving unit:

$$
v_{u}= \begin{cases}v_{f}, & \rho \leq \rho_{\max }, \\ v_{f}\left[1-\left(\frac{\rho-\rho_{\max }}{\rho_{\mathrm{jam}}}\right)^{\alpha}\right]^{\beta}, & \rho>\rho_{\max } .\end{cases}
$$

2.2.3. Vehicle Moving Model. In this paper, we use the classical model of medium traffic. In the section of the moving part, the speed of the vehicle moving is determined by the speed-density model, which is based on the fixed speed. The deceleration area, if there is no queuing, assumes that beginning $t$ from 0 , the vehicle is located in $z_{0}$; the vehicle at the time of $t(z)$ is defined as

$$
t(z)=\frac{z-z_{0}}{v_{u}} .
$$

Assume that the time is $t=0$, the vehicle is located in $z_{0}$, and then the position is

$$
z(t)=v_{u}+z_{0} .
$$

If there is a queue at the location $z(t)$, then the location of the vehicle at any time $t$ is given by the following formula:

$$
z(t)=e^{\lambda(t) t}\left(z_{0}+\frac{v_{u}}{\lambda(t)}\right)-\frac{v_{u}}{\lambda(t)},
$$

Where $\lambda(t)=\frac{-v_{u}}{q_{0}+l(c t-m)}$. 
2.3. Data Calibration Algorithm Based on RBF Neural Network. RBF neural network is the radial basis function (RBF) as a hidden unit "base," constituting a hidden layer, hidden layer to the input vector transform, which can realize the nonlinear mapping from input to output. From the geometric sense, it is equivalent to the given sparse samples point to restore a continuous hypersurface to the point; in the surface to meet the sample value, the network application is equivalent to the estimation of the unknown point value. The learning methods are as follows $[14,15]$.

Defining the network training sample input as $X_{k}$, the actual output of the $j$ output neurons is

$$
y_{k j}\left(X_{k}\right)=\sum_{i=1}^{N} w_{i j} \varphi\left(X_{k}, X_{i}\right), \quad j=1,2, \ldots, J .
$$

Among them, the Green function $\varphi\left(X_{k}, X_{i}\right)=G\left(X_{k}, X_{i}\right)$ is commonly chosen as the basic function; the objective function can be defined as $E=(1 / 2) \sum_{k=1}^{N} e_{k}^{2}, N$ is the number of training samples, and $e_{k}$ is the signal error, which is defined as $e_{k}=d_{k}-Y_{k}\left(X_{k}\right)=d_{k}-\sum_{i=1}^{I} w_{i} G\left(\left\|X_{k}-t_{i}(n)\right\|_{C_{i}}\right)$.

\section{Congestion Control Strategy}

3.1. Basic Hypotheses. In the two-way traffic network, the segments are subdivided into segments (Figure 4), and the segments have the same physical characteristics. Each section of the lower reaches its capacity constraints, known as the output capacity. The size of the output power depends on the physical characteristics of the road (such as the width and the slope) or unexpected events, control facilities, and so forth. The section upstream is a mixed traffic area, the area is mixed with the vehicles in each direction of traffic flow, the lower reaches of the area, and the vehicle runs in parallel and independent of each other. The drainage area is subdivided into three separate subregions, which are used for turning, left, straight, and right. In view of the path selection, traffic demand, road characteristics, and so on, this paper makes the following assumptions:

(1) The traffic between any OD pairs is constant. All paths are obtained by the literature, and they will not change their travel path because of the congestion, unless the traffic control is implemented.

(2) In the entire simulation time period, it uses the periodic boundary conditions.

(3) All intersections are defined as having the same input and output power, without any signal control, which only considers the deterministic component of the traffic flow.

(4) If a particular outlet (e.g., left) is congested, the traffic flow will be formed in the rear of the road section. If obstacles cannot be cleared in time, the line will eventually extend and cross over to the other lanes, causing traffic jams on the road.

3.2. Spatial Structure of Traffic Jam. If a traffic accident occurs in the traffic network, it might as well assume that a road from east to west in the network will cause a sharp drop in the traffic capacity of the road. When the vehicle line up to the intersection of the road, the plan is to turn left (from south to north) or turn right (north to south) into the road to form a new line of vehicles. Vehicle queue continues to spread until the upstream intersection and causes more road congestion. The distribution of traffic congestion on the network is given after a period of traffic accident. It can be seen that the traffic jam on the space structure is generally a diamond structure. But according to the latest research results show that there is no accident of two-way lattice network congestion space structure which is generally a central symmetrical graphics. After the accident, the traffic jam is extended from the accident to the tree structure, which is shown in the paper. If the accident lasts long enough, the range of Congestion Propagation in four directions to the four corners of the world will be roughly the same. This feature is crucial to ease traffic congestion, because these sections for jam provide a buffer zone.

In order to show the growth process of congestion, Figure 1 depicts the conditions where there is no artificial intervention in the Congestion Propagation tree. Figure 1 (left) shows the process of the accident which removes the previous congestion growth process. It can clearly see the scale of the congestion and the network in which each section of the road is in a congested state of the total time. Figure 1 (right) shows the formation of a closed loop. When the accident lasts long enough, the stop line width is divided into an unreasonable (turning direction allocation), and the closed loop is formed in the process of the traffic accident relief. Once the incident is cleared, the vehicle starts and generates the start wave. At the same time, the upper reaches of the vehicle continue to enter the congested area. At first, a straight line of vehicles can effectively leave the congestion area, while the queue also looks to start to dissipate and then turn the vehicle quickly to get to the end of the initial line and line up [16]. Due to the distribution of the direction of the traffic flow direction of the car park, the traffic flow direction is insufficient, which causes that the turning vehicles cannot leave the congestion area in time. Sometime later, straight line direction of the traffic flow is affected by the turning of the vehicle and fewer vehicles are pulled out of the jam area, while the arrival of regional congestion of vehicles is increasing. This has led to a growing traffic congestion. Without manual intervention, congestion will continue to grow until the entire network is covered.

3.3. New Congestion Control Strategy. In a one-way street grid, the exit of a section of the road is divided into two directions: straight and turning (left or right). In this form of traffic, the vehicle can be used to restrict the area of the vehicle entering the area by using the measures to prohibit the use of a straight line or a turn. In the two-way traffic network, the export of road segments needs to be divided into three directions: turn left, go straight, and turn right. For the same section, not only can the straight line of the vehicle enter the congested area, but also left or right turn vehicle can also enter the congested area. In this form of transportation, the simple 


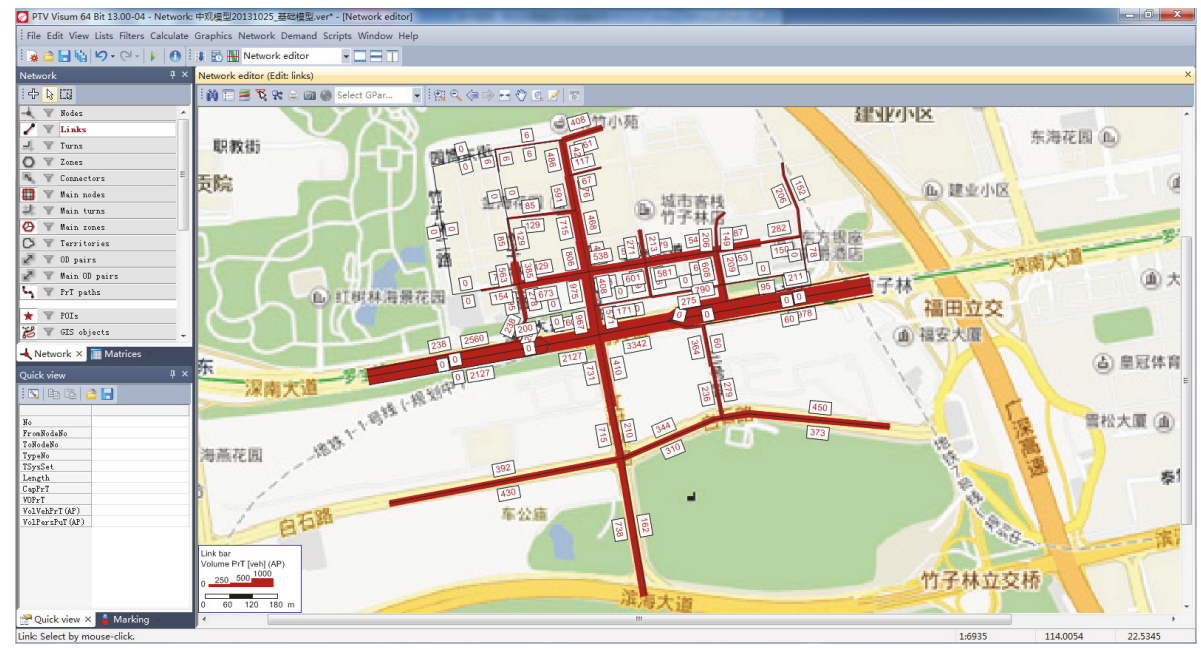

FIGURE 2: Flow distribution of Zhuzilin area roads.

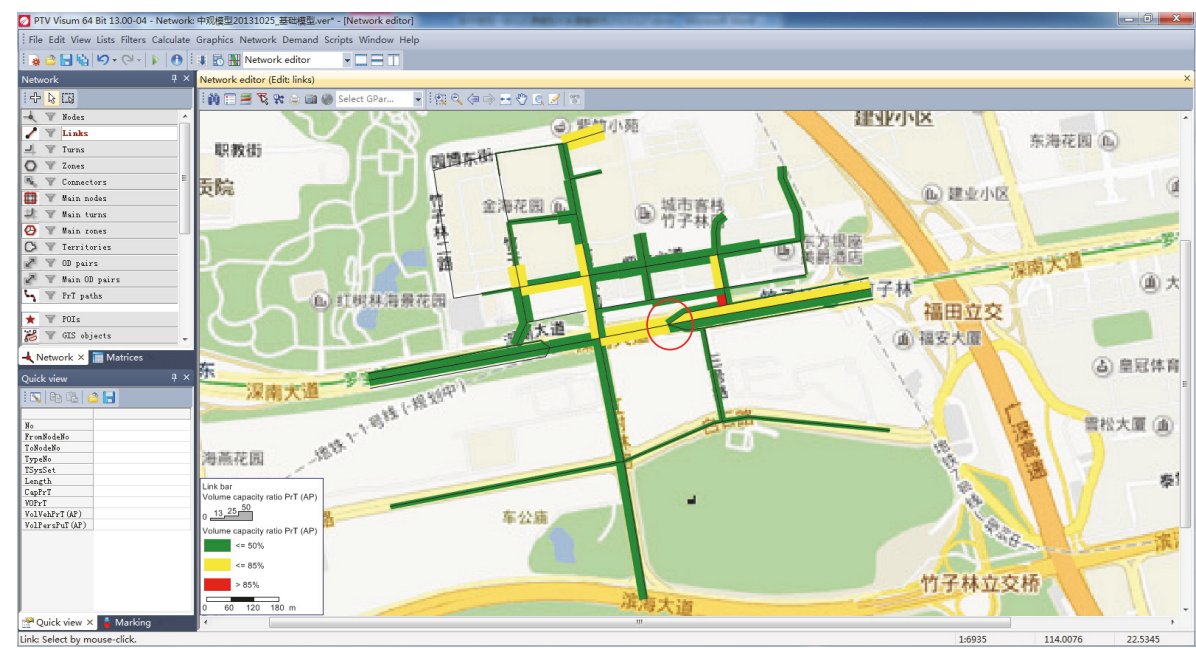

FIgURE 3: Regional road saturation distribution.

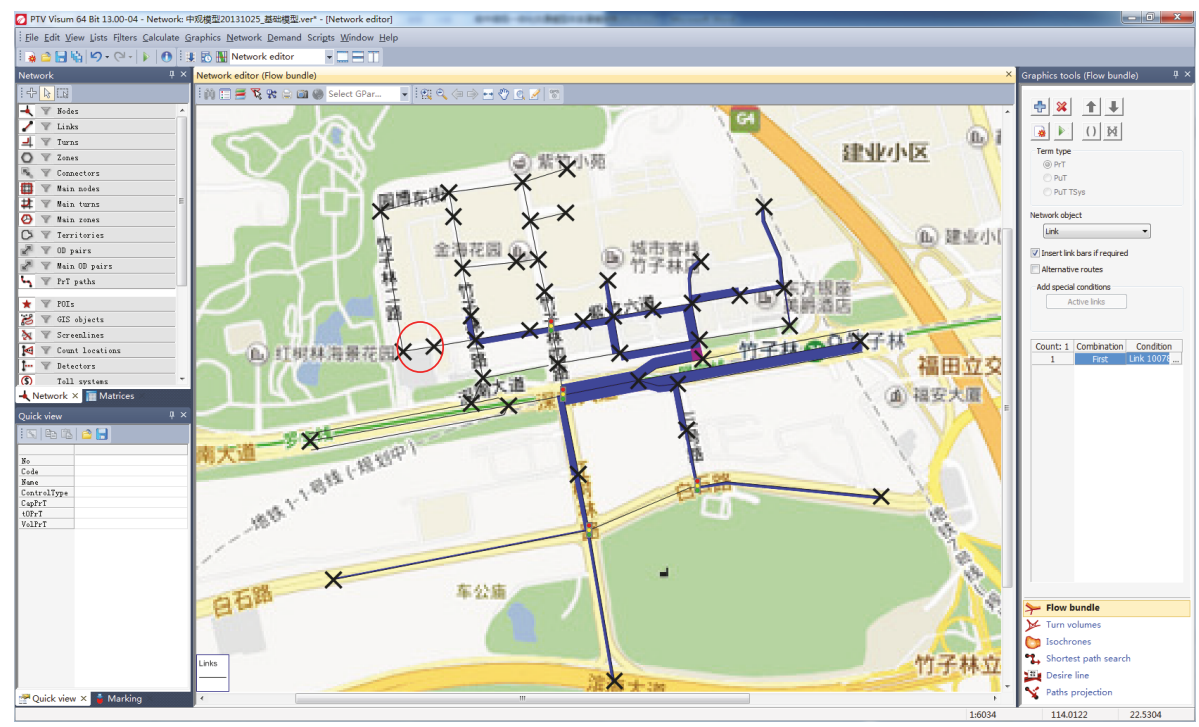

FIgURE 4: Road congestion cobweb diagram. 


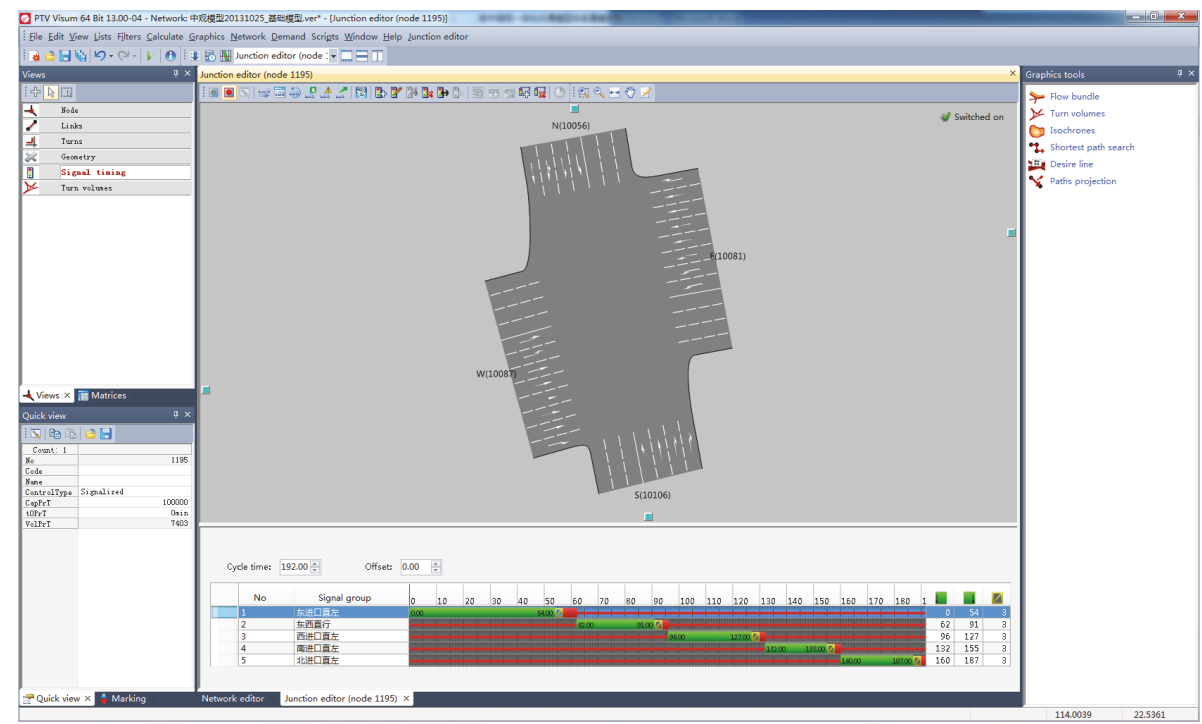

Figure 5: Current situation of the intersection of the channel control scheme.

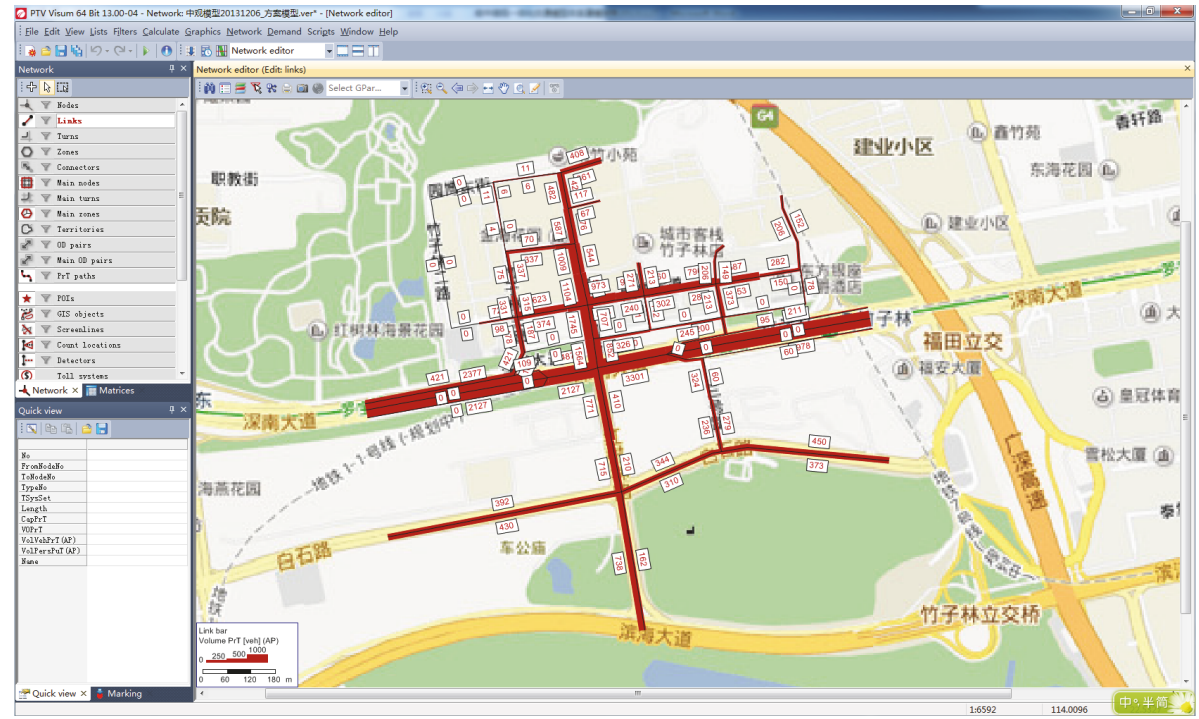

Figure 6: Road traffic distribution (Zhuzilin area modify credit control scheme).

use of a ban on the use of direct line of measures cannot effectively limit the congestion area of the vehicle to enter the congested area. Thus, the existing congestion control strategy for a one-way street network can not be completely copied to the two-way traffic network [17].

In this paper, the congestion control strategy is to turn prohibition and the vehicles intelligent behavior, and in the periphery of the congested area a vehicle prohibition area is established, while the vehicle changes its route in congested area to take a temporary path. According to the shape of the congestion, the control strategy is presented as follows: (1) single track control strategy, (2) multiline control strategy, (3) control strategy, and (4) tree control strategy.

The fourth kind of control strategy is proposed in this paper that the formation of congestion is not only concerned with the site of the incident and traffic network structure and other factors are inseparable, but two-way lattice network, Congestion Propagation, should be in the path that the accident site is in the OD of the shortest path, whose shape is similar to the tree branches. So it is necessary to execute a variety of intelligent or artificial control measures in the periphery of the structure of congestion; hence the name is the tree control strategy $[12,18-20]$. Of course, this is no longer a single restriction; there is also induction of realtime path selection behavior. In the medium traffic model, the measures are forbidden to turn and real-time route choice in order to ease the traffic congestion. In the paper, the vehicle is used to cancel the condition that if the traffic congestion at the lower reaches the accident site which is completely dissipated, all the measures will be cancelled immediately. 


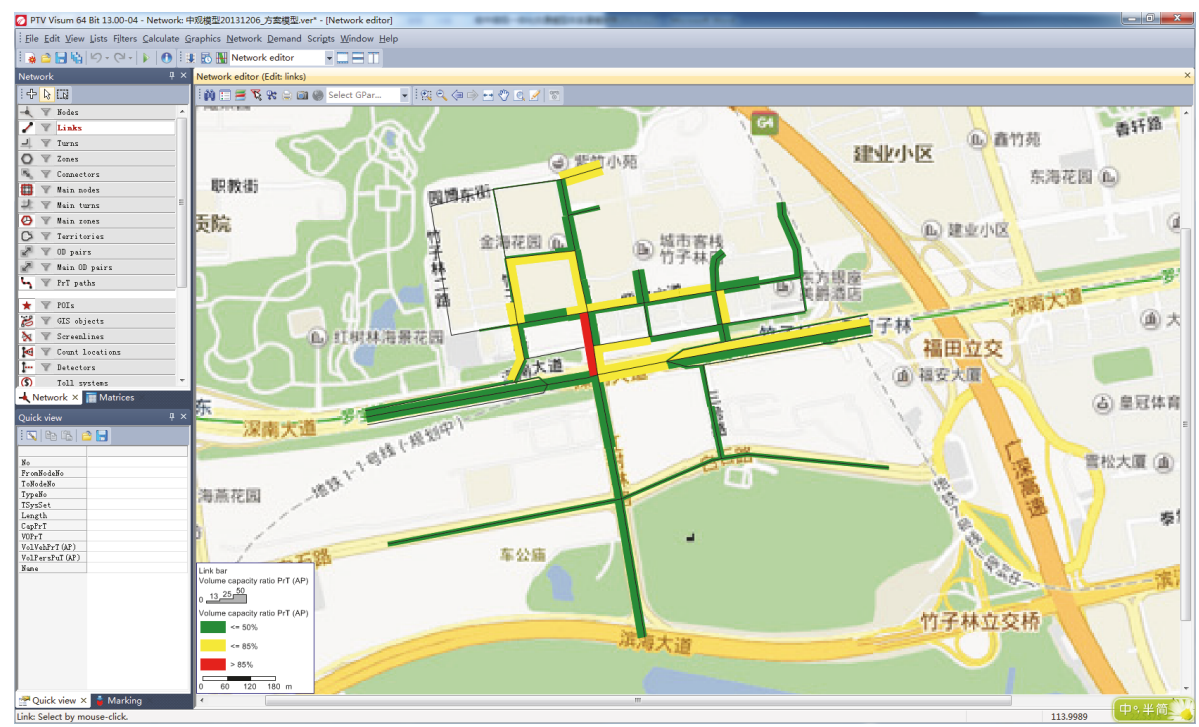

FIGURE 7: Road traffic distribution (Zhuzilin area modify credit control scheme).

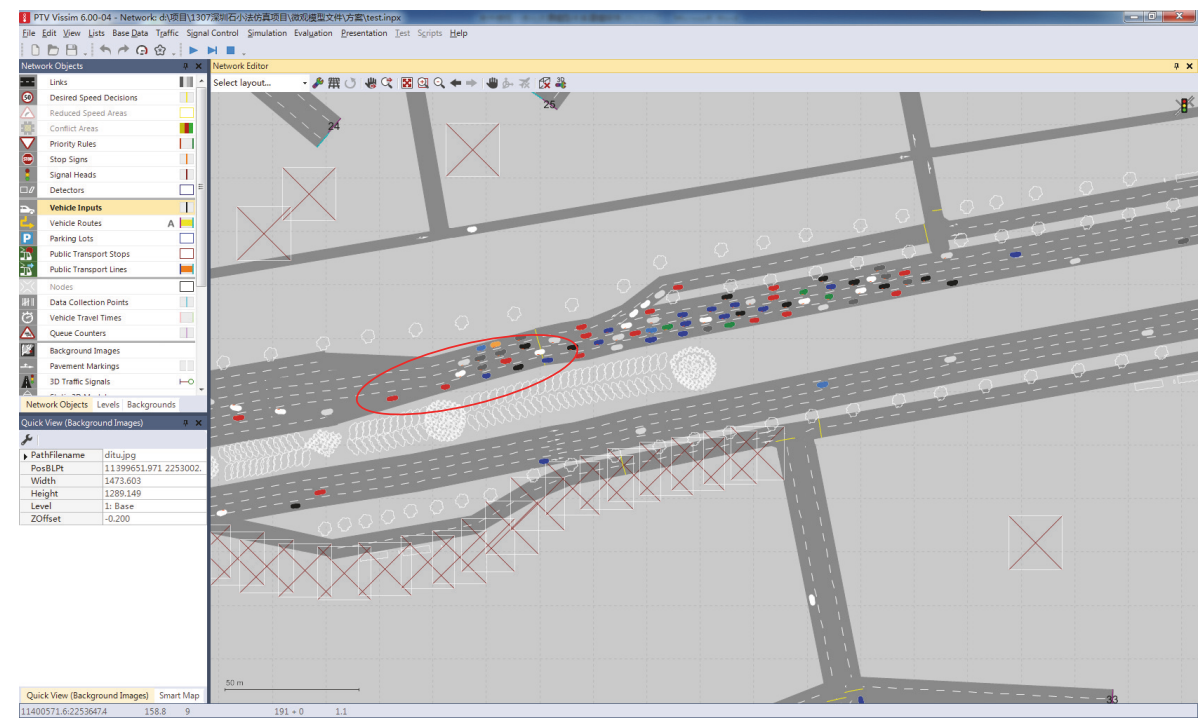

FIGURE 8: Vehicle queuing situation diagram.

\section{Case Study}

In this paper, we observe the average density flow rate and other indicators in the above conditions, so as to judge the congestion of the network. Secondly, the OD is set up, the OD points are defined, and the fourth kind of congestion dissipation strategies are adopted. Average density flow rate and other indicators are then analyzed. The accident occurred at the beginning of the accident upstream, if the entire simulation time is not to take any measures, then the queue continues to spread. According to the concept of traffic model, the traffic flow and saturation Zhuzilin area distribution are as shown below. From the perspective of traffic demand and traffic supply, the medium traffic model is to analyze the causes of traffic congestion and to analyze the congestion control scheme. In this study, the Zhuzilin area is used to establish an example of a mesoscopic traffic model for congestion causes analysis and to make recommendations for improvement. Because this project is mainly integrated model of the technical analysis method research, the results may be different from the actual traffic conditions.

4.1. Cause Analysis of Congestion. According to the concept of traffic model, the traffic flow and saturation Zhuzilin area distribution are shown in Figures 2-6.

As can be seen from Figure 3, the Zhuzilin area is the main point of congestion on Zhuzilin Road, specifically, the six roads leading to the Shennan Boulevard Voeux Road (circled in red) sections. Using the Visum flow bundle function, the view of the road traffic situation is shown in Figure 4. 


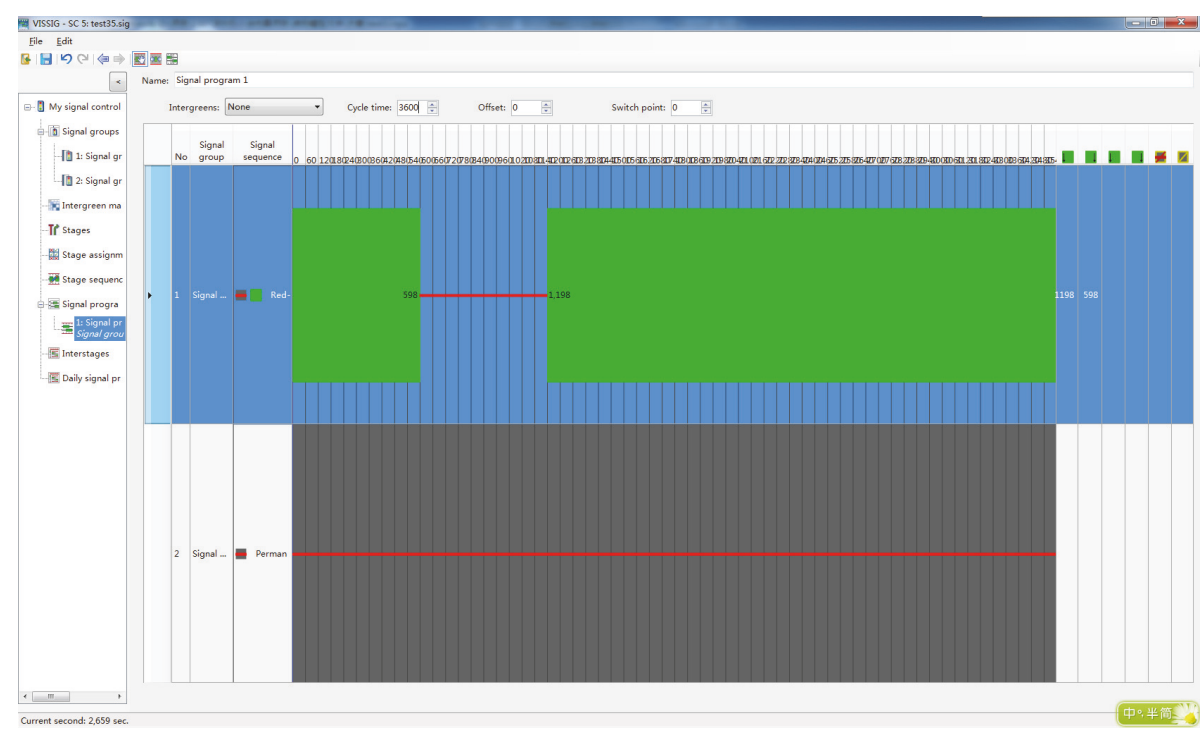

FIGURE 9: Signal lamp setup diagram.

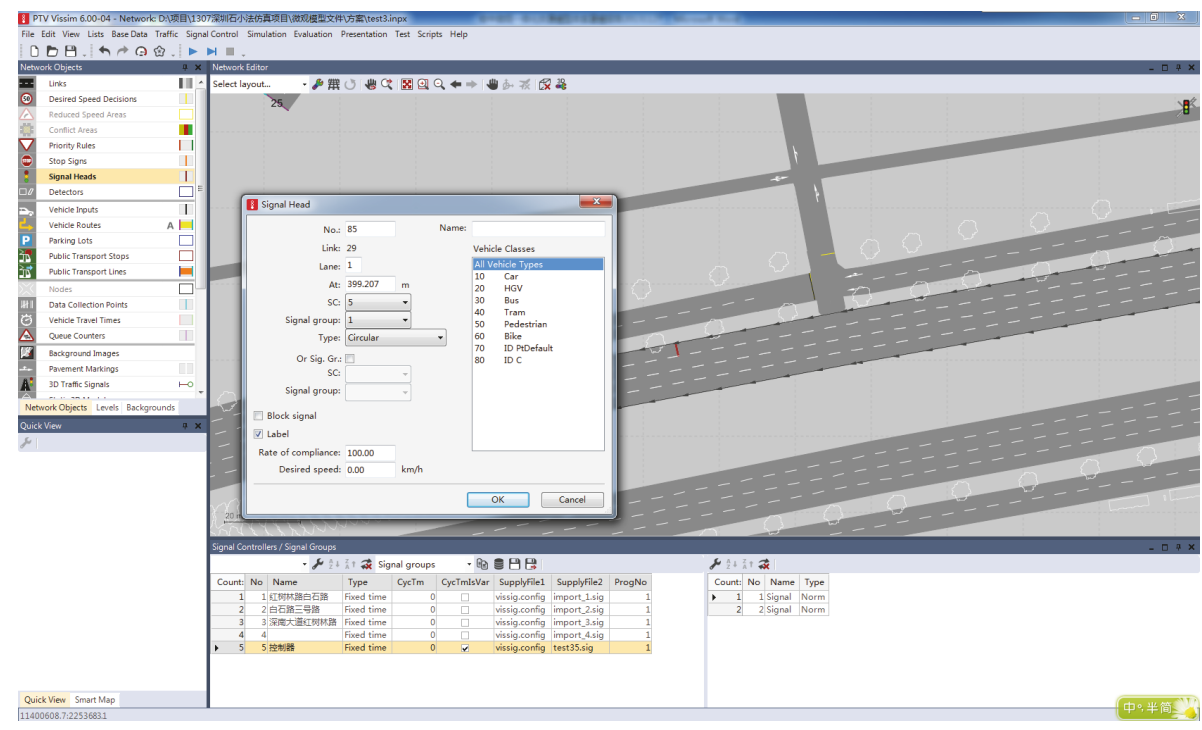

FIGURE 10: Signal lamp setup diagram.

The spider web analysis diagram shows that this section of the traffic is mainly to the Shennan Road East of the Uturn car flow, which has a part of the traffic flow or through the northwest corner of the intersection of Shennan AvenueMangrove Road (pictured in red circle location) leading to the Shennan Road East. This part of the car flow is from four Zhuzilin Road left turn lanes into Shennan Avenue. We use flow bundle function and check the traffic situation of the four Zhuzilin Road left turn lanes into Shennan Avenue.

It is found that the traffic from the four Zhuzilin left turn lanes into Shennan Road traffic is large. Therefore, the Shennan Avenue-Mangrove Road intersection signal control and channelization schemes were studied. It is found that the north entrance of the left turn traffic green signal ratio is only
0.14. According to the investigation of the saturation current of the intersection, the distance of the head is 2.53 seconds; that is, the saturation capacity of single lane is $1400 \mathrm{pcu} / \mathrm{h}$. From this calculation, the actual turning capacity is $2.5 *$ $1400 * 0.14=490 \mathrm{pcu} / \mathrm{h}$. At the same time, Shennan Road East imports the leftmost lane for left U-turn lane (due to the presence of two dedicated left turn lanes, the survey found that the lane is $80 \%$ of the traffic to U-turn car flow), and the green signal ratio is 0.28 , which is calculated to get the actual turnover capacity for $0.8 * 1400 * 0.28=310 \mathrm{pcu} / \mathrm{h}$. When the volume from the four Zhuzilin left turn lanes into Shennan Road traffic is excessive, part of the traffic flow opts to reach Zhuzilin Road via the six lanes of Shennan Avenue after making a U-turn after traveling east, which is shown in Figure 5. 


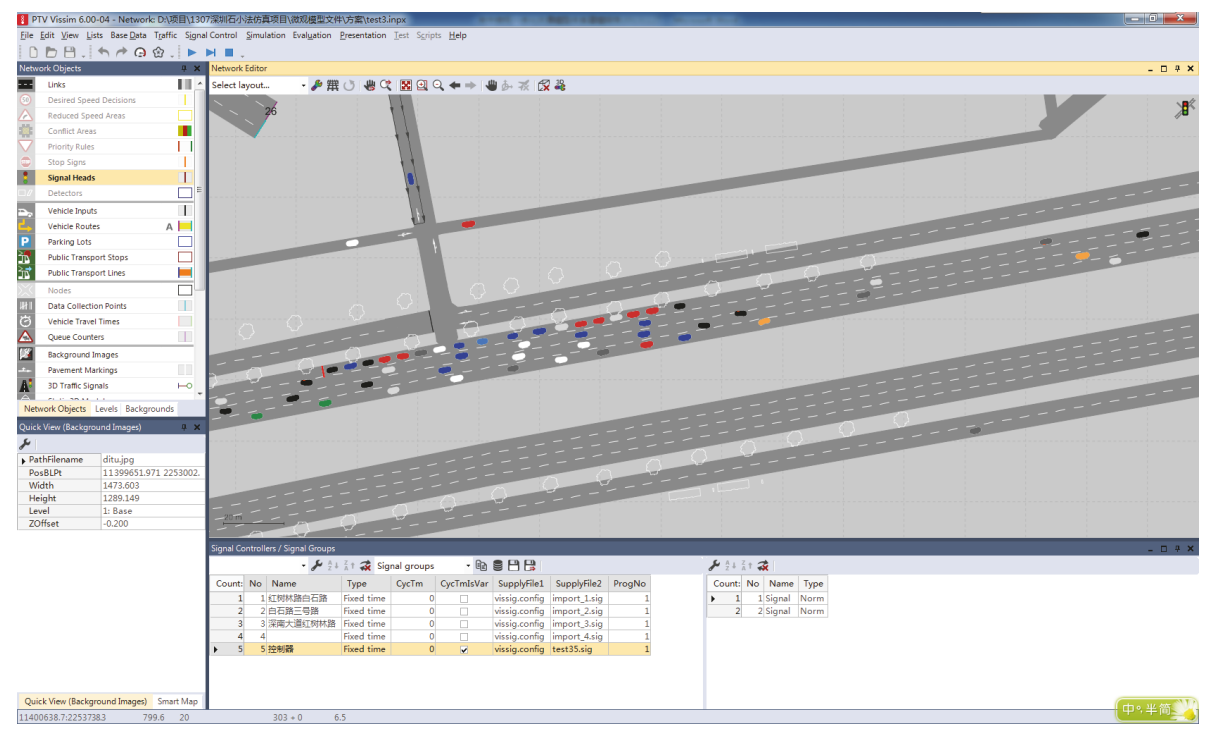

FIGURE 11: Queue start chart.

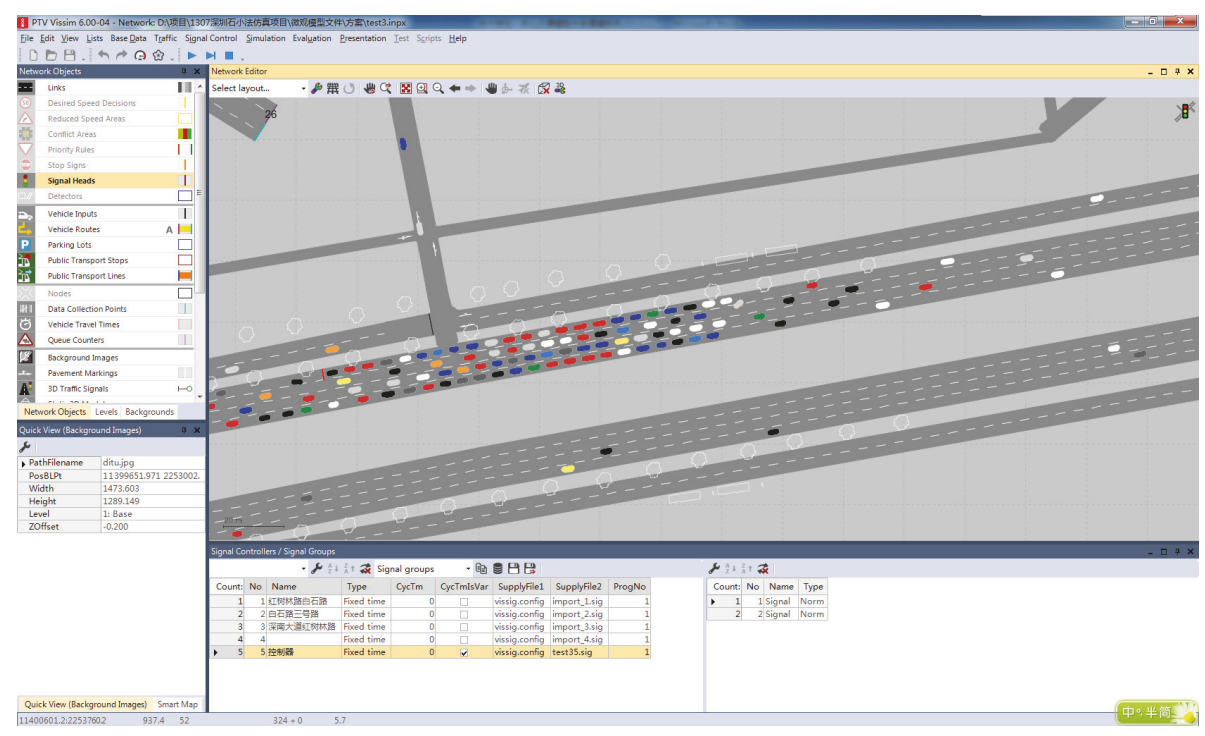

FIGURE 12: Queuing growth process.

Thus, the main cause being the four Zhuzilin left lanes overloading Shennan Road's traffic capacity is insufficient. According to the above reasons, to improve the design of Zhuzilin four Shennan Road, it is necessary to increase the capacity of left turn including the increase of the steering drive and improving the green letter. Considering that there are already 2.5 left turn lanes, the proposed method is proposed to improve the green letter ratio. Also, the fourth control strategy is used.

Thus, the calculated regional traffic flow and saturation distribution are shown as Figures 6 and 7.

It can be seen from Figure 6 and 7 that the improved scheme can be significantly decreased by routing six lanes of traffic from Zhuzilin Road into Shennan Boulevard des Voeux Road traffic; the traffic will choose four Zhuzilin
Road left turn lanes into Shennan Avenue. At the same time, however, we also found that the congestion of the four Zhuzilin Road turn lanes increased, which requires further analysis, which we have deferred.

4.2. Microscopic Traffic Model. Microscopic traffic model is based on the driving of vehicles in the road network and analyzes the bottleneck of the traffic congestion, and the improvement of the bottleneck points is analyzed [14]. In this section, for project established using the Zhuzilin area microscopic traffic model, for example, the congestion bottleneck analysis and process improvement measures are presented. Because this project is mainly integrated model of the technical analysis method research, the results may be different from the actual traffic conditions. 


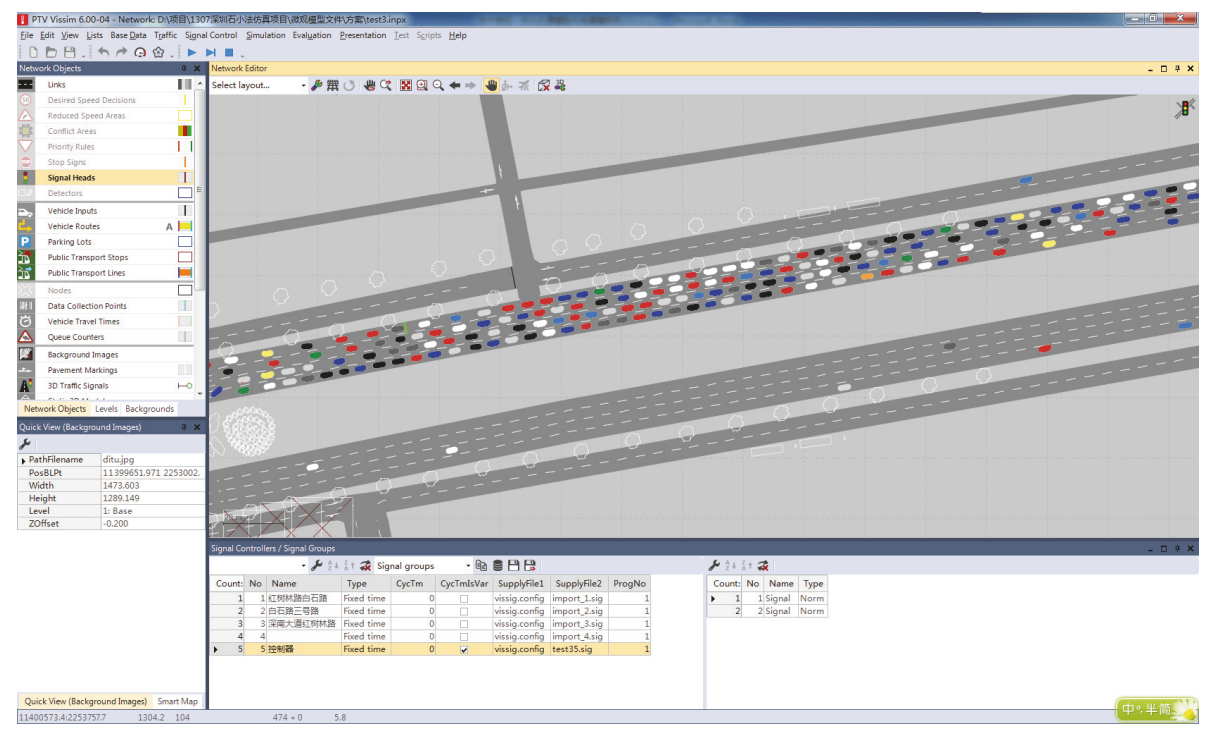

FIGURE 13: Accident handling line began to evacuate.

As shown in the Figure 8, the location of the congestion bottleneck through the vehicle queue is determined.

Analysis of the queuing situation is due to the formation of the main road, and the middle section is the bottleneck of the whole road traffic. Microscopic traffic model can be used to analyze the causes of sudden traffic congestion and traffic congestion, to analyze the feasibility of the scheme, and to evaluate the effect of different traffic congestion on the traffic flow. The simulation of the emergency traffic congestion is analyzed. The microscopic traffic model analysis method used in the traffic congestion is similar to that used in the analysis of the traffic congestion. Therefore, in this section, for the project established using the Zhuzilin area microscopic traffic model, for example, the congestion response speed and evacuation analysis process are discussed.

Because this project is mainly integrated model of the technical analysis method research, the results may be different from the actual traffic conditions. In the microscopic model, an accident caused by a road bypass signal lamp is simulated. For example, we have to take 10 minutes to complete the accident, and the accident occurred in the tenth minutes. The signal light can be then synchronized with the red light for about $600 \mathrm{~s}$, until about $1200 \mathrm{~s}$, when it is synchronized with the green light, as shown in Figures 9-13.

\section{Conclusion}

Traffic incident is one of the main reasons leading to the traffic jam. In this paper, the traffic congestion caused by accidents is simulated by using the improved medium traffic model, and the propagation law of traffic congestion is found. The control strategy is put forward for the first time. The simulation results also prove that the control strategy proposed in this paper is effective and feasible. According to the temporal and spatial evolution of the paper, we can see that the network has been improved on the whole. But the paper is still showing many irregularities, such as not considering the use of advanced intelligent transportation methods for congestion control, cross-signaling intersection control, and information guidance. Also not considered was the use of more advanced traffic prediction methods, informed in advance by dynamic network information, and antiblocking, as well as the effective combination of the above-mentioned methods. This is also the direction of future efforts of the author.

\section{Conflicts of Interest}

The authors declare that there are no conflicts of interest regarding the publication of this paper.

\section{Acknowledgments}

This study was supported by the Guangdong Natural Science Fund (2015A030313626), Special Fund of Frontier and Key Technology Innovation of Guangdong Province (Provincial Major Science and Technology Project) (2014B010112008, 2015B010106001, 2015B010129003, and 2016B010109007), Pearl River S\&T Nova Program of Guangzhou (201610010034), Guangdong Science and Technology Program (2013B01010201, 2013B010102018, and 2013B090600152), Guangzhou Science and Technology Project (no. 2014Y2-00044), National Natural Science Foundation of China (61603105), and Scientific Research Project of Guangzhou Municipal Colleges and Universities (1201610154).

\section{References}

[1] M. Junghare and M. Shimpi, "A survey of congestion control protocols for wireless sensor network," in Proceedings of the IJCA Proceedings on National Conference, vol. 43, 2012. 
[2] J. Wan, X. Xu, R. Feng, and Y. Wu, "Cross-layer active predictive congestion control protocol for wireless sensor networks," Sensors, vol. 9, no. 10, pp. 8278-8310, 2009.

[3] O. Jahn, R. H. Möhring, A. S. Schulz, and N. E. Stier-Moses, "System-optimal routing of traffic flows with user constraints in networks with congestion," Operations Research, vol. 53, no. 4, pp. 600-616, 2005.

[4] C. Yang, X. Wang, Z. Li, Y. Li, and C. Su, "Teleoperation control based on combination of wave variable and neural networks," IEEE Transactions on Systems, Man, and Cybernetics: Systems, vol. 47, no. 8, pp. 2125-2136, 2017.

[5] C. Yang, Z. Li, and J. Li, “Trajectory planning and optimized adaptive control for a class of wheeled inverted pendulum vehicle models," IEEE Transactions on Cybernetics, vol. 43, no. 1, pp. 24-36, 2013.

[6] C. Yang, X. Wang, L. Cheng, and H. Ma, "Neural-learningbased telerobot control with guaranteed performance," IEEE Transactions on Cybernetics, vol. 47, no. 10, pp. 3148-3159, 2017.

[7] Z. Zhao, Y. Liu, and F. Luo, "Output feedback boundary control of an axially moving system with input saturation constraint," ISA Transactions, vol. 68, pp. 22-32, 2017.

[8] Z. Zhao, Y. Liu, F. Guo, and Y. Fu, "Vibration control and boundary tension constraint of an axially moving string system," Nonlinear Dynamics, vol. 89, no. 4, pp. 2431-2440, 2017.

[9] Z. Zhao, Y. Liu, and F. Luo, "Boundary control for a vibrating string system with bounded input," Asian Journal of Control, In press.

[10] Z. Zhao, Y. Liu, W. He, and F. Luo, "Adaptive boundary control of an axially moving belt system with high acceleration/deceleration," IET Control Theory \& Applications, vol. 10, no. 11, pp. 1299-1306, 2016.

[11] Z.-J. Zhao, Y. Liu, F. Guo, and Y. Fu, "Modelling and control for a class of axially moving nonuniform system," International Journal of Systems Science, vol. 48, no. 4, pp. 849-861, 2017.

[12] W. K. Newey, "Nonparametric continuous/discrete choice models," International Economic Review, vol. 48, no. 4, pp. 1429-1439, 2007.

[13] S. Chen and N. Yang, "Congestion avoidance based on lightweight buffer management in sensor networks," IEEE Transactions on Parallel and Distributed Systems, vol. 17, no. 9, pp. 934-946, 2006.

[14] D. Howard, B. Mark, and H. Martin, Neural Network Toolbox For Use with Matlab, The MathWorks, Natick, Mass, USA, 2007.

[15] N. F. Zhang, J. F. Yang, Y. J. Xue, Z. Li, and X. L. Huang, "Agricultural machinery operation posture rapid detection intelligent sensor calibration method based on RBF neural network," Applied Mechanics and Materials, vol. 373-375, pp. 932-935, 2013.

[16] A. Petrin, "Quantifying the benefits of new products: the case of the minivan," Journal of Political Economy, vol. 110, no. 4, pp. 705-729, 2002.

[17] S. Peeta and A. K. Ziliaskopoulos, "Foundations of dynamic traffic assignment: the past, the present and the future," Networks and Spatial Economics, vol. 1, no. 3-4, pp. 233-265, 2001.

[18] Z. Zhao, X. Wang, C. Zhang, Z. Liu, and J. Yang, "Neural network based boundary control of a vibrating string system with input deadzone," Neurocomputing, 2017.

[19] W. He, Y. Chen, and Z. Yin, "Adaptive neural network control of an uncertain robot with full-state constraints," IEEE Transactions on Cybernetics, vol. 46, no. 3, pp. 620-629, 2016.
[20] W. He, Y. Dong, and C. Sun, "Adaptive neural impedance control of a robotic manipulator with input saturation," IEEE Transactions on Systems, Man, and Cybernetics: Systems, vol. 46, no. 3, pp. 334-344, 2016. 


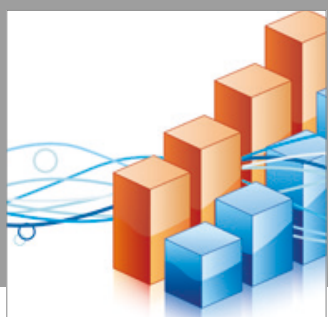

Advances in

Operations Research

vatersals

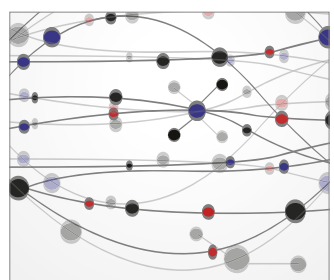

\section{The Scientific} World Journal
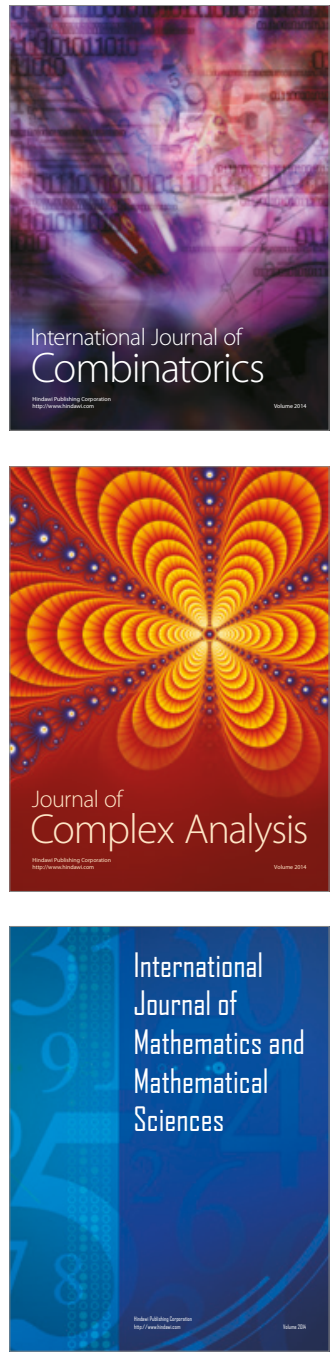
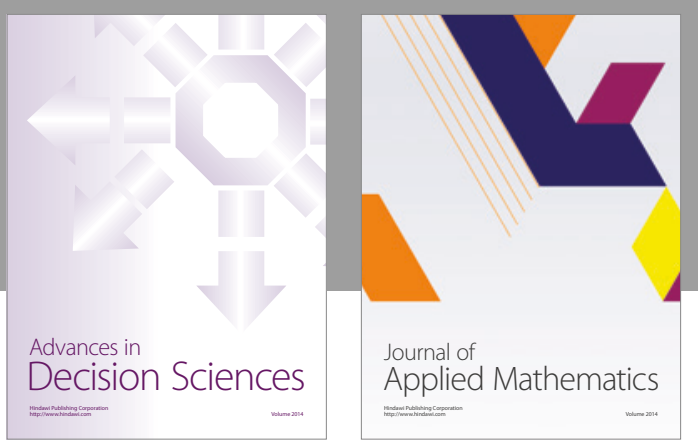

Algebra

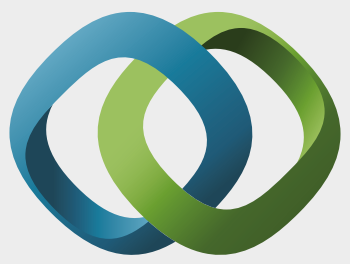

\section{Hindawi}

Submit your manuscripts at

https://www.hindawi.com
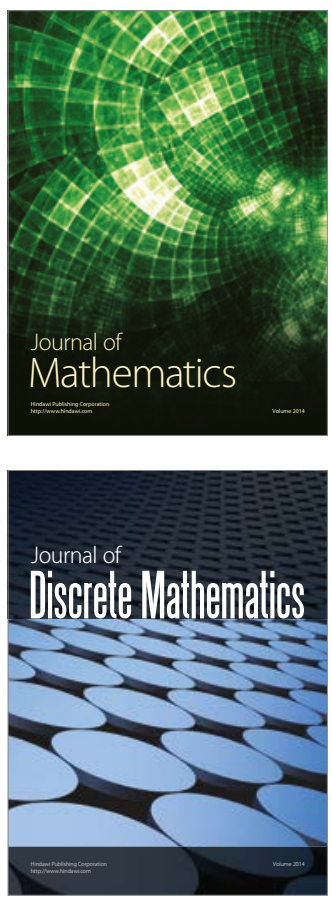

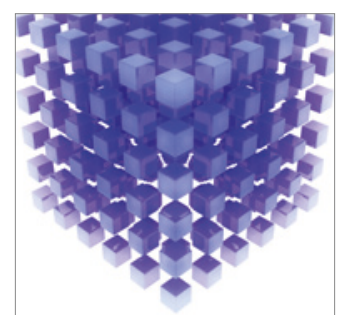

Mathematical Problems in Engineering
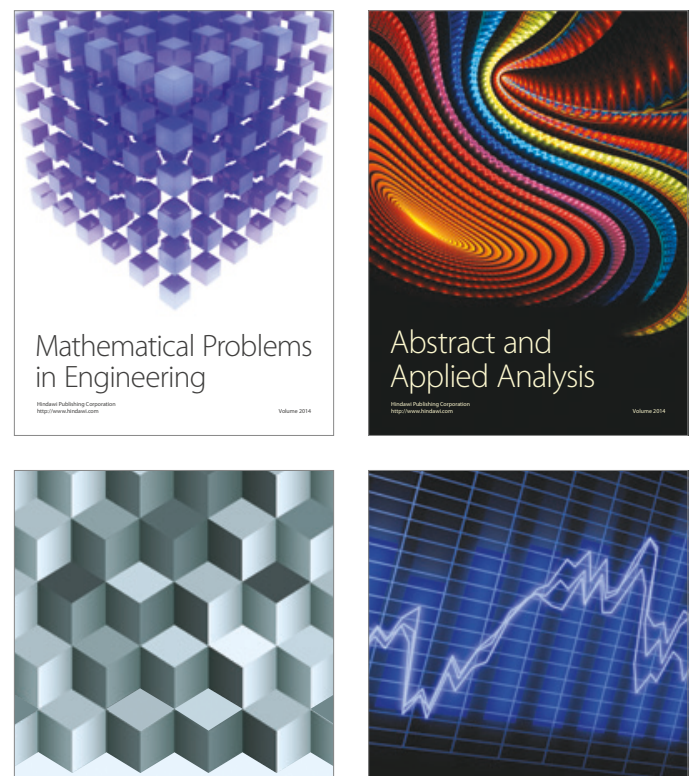

Journal of

Function Spaces

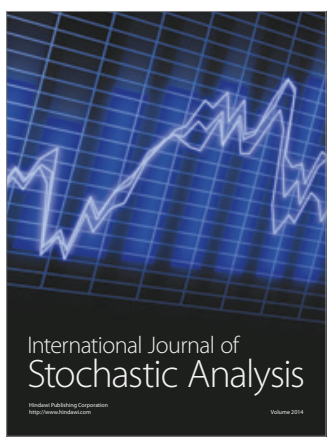

Probability and Statistics
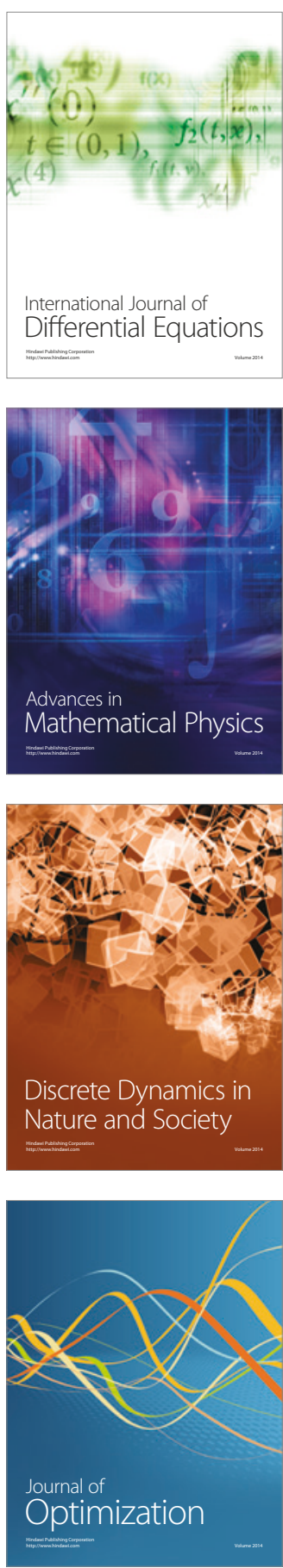